\title{
Behavioral and Neurochemical Vulnerability During Adolescence in Mice: Studies with Nicotine
}

\author{
Walter Adriani', Oleg Granstrem ${ }^{2,3}$, Simone Macri', Galina Izykenova ${ }^{2,3}$, Svetlana Dambinova ${ }^{2,3}$ and \\ Giovanni Laviola*,I
}

'Section of Behavioral Pathophysiology, Department of Cell Biology and Neuroscience, Istituto Superiore di Sanita', Roma, Italy; ${ }^{2}$ Department of Neurology and Neurosurgery, 'IP Pavlov' State Medical University, St Petersburg, Russia; ${ }^{3}$ Chemistry Department, Emory University, Atlanta, GA, USA

\begin{abstract}
People are very likely to start psychoactive drug use during adolescence, an earlier onset being associated with a higher risk of developing addiction later in life. In experiment I, Pre- (postnatal day (pnd) 23-35), Mid- (pnd 36-48), or Post- (pnd 49-6I) adolescent mice underwent a restricted-drinking period $(2 \mathrm{~h} /$ day for 12 days), one bottle containing water and the other containing nicotine ( $10 \mathrm{mg} / \mathrm{l})$ or water. After this period, Mid-adolescents showed prominent exploration and reduced anxiety in the plus-maze. This ontogenetic profile was dampened by nicotine consumption. After 2 months, these mice were tested in a novel environment ( 30 min/day for 3 days). Locomotor-habituation profiles were specifically disrupted by nicotine consumption during Mid-adolescence, suggesting this age as a critical period. In experiment II, Mid-adolescent (pnd 35-44) and adult (pnd > 70) mice were pretreated with nicotine (0, 0.03, 0.10, $0.30 \mathrm{mg} / \mathrm{kg} /$ day for 10 days). Acute nicotine administration had opposite effects on anxiety in adolescents and adults. At 2 months after pretreatment, we measured levels of AMPA GluR2/3 subunits, thought to be involved in the control of addictive behaviors. Nicotine exposure during Mid-adolescence dose-dependently downregulated these subunits in the striatum and hippocampus, but comparable exposure during adulthood had either opposite or no effects. NMDA NR2A/B subunits were affected by nicotine, but without agerelated differences. The present data identified a nicotine-vulnerable age window, characterized by long-term disruption of locomotor habituation and downregulation of AMPA receptors. These findings support neurobiological vulnerability to drugs in adolescent humans. Neuropsychopharmacology (2004) 29, 869-878, advance online publication, 10 December 2003; doi: I 0.1038/sj.npp. 1300366
\end{abstract}

Keywords: nicotine; vulnerability; plus-maze; addiction; adolescence; AMPA receptors; immunoreactivity; mice

\section{INTRODUCTION}

Adolescence is associated with increased sensation-seeking, reckless and risk-taking behaviors, low levels of harm avoidance and anxiety (Arnett, 1992; Wills et al, 1994). The age of first encounter with psychoactive compounds is critical, since a higher probability to shift from use to abuse and to develop addiction is associated with an early approach (Robins and Przybeck, 1985; for tobacco, see Taioli and Wynder, 1991; Anthony and Petronis, 1995; Breslau and Peterson 1996). Nearly one-third of worldwide adults are smokers, and the majority started this habit when adolescents (Mansvelder and McGehee, 2002). Adolescents also express symptoms of nicotine dependence after only few cigarettes (DiFranza et al, 2000).

\footnotetext{
*Correspondence: G Laviola, Behavioral Pathophysiology, Lab Fisiopatologia OS, Istituto Superiore di Sanita', viale Regina Elena 299, I-00 I 6 I Roma, Italy, Tel: + 39064990 2105, Fax: + 39064957821 ,

E-mail: laviola@iss.it

Received I I July 2003; revised 28 October 2003; accepted 3 I October 2003

Online publication: 3 November 2003 at http://www.acnp.org/ citations/Npp I I 030303307/default.pdf
}

A suitable animal model is available for laboratory studies. Periadolescence is classically defined in rodents as the ontogenetic period including the week preceeding the onset of puberty and few days thereafter (Spear and Brake, 1983). During this period, brain areas show proliferation and maturation of axon terminals and synapses (Stamford, 1989; Teicher et al, 1995). Adolescent rodents show elevated levels of novelty seeking (Adriani et al, 1998), impulsivity (Adriani and Laviola, 2003), and risk-taking behavior (Macri' et al, 2002), as well as reduced response to stress (Adriani and Laviola, 2000). Oral intake of alcohol (Ferris et al, 1998) and nicotine (Adriani et al, 2002) is elevated. Recent studies indicate dramatic differences in response to nicotine between adolescent and adult rats (Faraday et al, 2001), enhanced vulnerability to develop nicotine addiction being found around puberty (Adriani et al, 2003). Experimental interest for the neural effect of adolescent nicotine exposure is growing (Trauth et al, 1999, 2001). Investigation of molecular consequences of early-onset nicotine addiction is important to elaborate intervention strategies, since tobacco use during adolescence could be a 'gateway' for other drugs of abuse (Kandel et al, 1992; Torabi et al, 1993). 
The pathogenic process leading to addiction is so far not completely understood. The rewarding power of abused drugs is classically ascribed to meso-limbic dopaminergic projections (Wise, 1996; Robbins and Everitt, 2002). The onset of addictive behavior also implies alterations in the habit-forming process, which is thought to rely upon the nigro-striatal projections (Gerdeman et al, 2003). Glutamate receptors may play an important role in this regard (Gerdeman et al, 2003). In turn, nicotine is able to act on meso-limbic and nigro-striatal neurons that project to striatum, amygdala, and prefrontal cortex (Picciotto et al, 1998), thus modulating anxiety, behavioral (dis)inhibition, reward, and habit-forming processes. Nicotine may act not directly on dopamine cell bodies, but rather through glutamatergic inputs to dopamine neurons (Dajas-Bailador et al, 2000; Prendergast et al, 2001; Meyer et al, 2002; Mansvelder and McGehee, 2002). Recently, it was demonstrated that AMPA receptors play a key role in pavlovian approach to drug cues and in cocaine-seeking behavior in rats (DiCiano et al, 2001; DiCiano and Everitt, 2001; Sutton et al, 2003). Specifically, the glutamatergic input to the striatum might affect neuronal plasticity and habit-forming processes (Winder et al, 2002; Fasano and Brambilla, 2002; Gerdeman et al, 2003), which are key processes for the onset of drug addiction (Robbins and Everitt, 2002; Nestler, 2001, 2002). Even if molecular mechanisms are not yet clear, a pathological decrease of the AMPA glutamatergic input in the striatum might be considered an important hint for plastic changes leading to addictive habits.

The present study focused on the effects of nicotine on anxiety and exploration in the elevated plus-maze. We also investigated the role of nicotine exposure during adolescence in the long-lasting neurobehavioral changes involved in vulnerability to addiction. Different aspects of vulnerability to nicotine during adolescence were assessed, using either a behavioral or a neurobiological approach. In a first experiment, we investigated the long-term behavioral effects of spontaneous nicotine intake during three age periods of adolescence (Pre- (postnatal day (pnd) 23-35), Mid- (pnd 36-48), Post- (pnd 49-61)). This experiment confirmed that vulnerability is specific of Mid-adolescence, and is not found at younger or older ages. Hence, in a second experiment, we assessed the long-term neurochemical consequences of nicotine exposure during Mid-adolescence, when compared to similar levels of drug exposure in already adult subjects. We demonstrated that the neurochemical effects of nicotine differ considerably depending on the age of exposure.

Some words must be spent about the two different nicotine-exposure paradigms adopted here. Both have their merits and possible shortcomes. The experimenter-imposed drug administration is a classical pharmacological tool, aimed at assessing the drug effects per se, independently of animal will. However, some stress associated with the handling and injection procedures is intrinsic and cannot be avoided. Conversely, the oral self-administration approach is perhaps more close to reality, in that animals can titrate the drug intake up to their individual satiation level. However, drinking levels can differ among subjects, and hence the interpretation of results may be difficult compared to 'pure' pharmacological assessments. The aim of the present work was not to directly compare the two paradigms, rather we adopted for each experiment the approach whose features were better fitting the actual purpose. Hence, to study nicotine effects on exploration and habituation, we chose oral intake, since satiation of drug appetite might be crucial to interfere with the natural drive toward exploration and novelty. Conversely, to investigate effects on glutamate receptors, a classical pharmacology approach was preferred, all animals receiving the same drug dosage. Indeed, a disomogeneous level of drug exposure might differentially affect receptor up/downregulation in single subjects, possibly leading to inconsistent results.

\section{MATERIALS AND METHODS}

\section{Subjects, Breeding, and Rearing Conditions}

Mice of the outbred CD-1 strain, without prior breeding experience, were purchased from a commercial breeder (Charles River Italia). On arrival, mice were housed in an air-conditioned room (temperature $21 \pm 1^{\circ} \mathrm{C}$, relative humidity $60 \pm 10 \%$ ), with a reversed 12-h light-dark cycle (light on at 2100). Water and food (Enriched Standard Diet, Mucedola, Settimo Milanese, Italy) were available ad libitum. After 2 weeks, breeding pairs were formed and housed in Plexiglas cages $(33 \times 13 \times 14 \mathrm{~cm})$, with metal tops and a sawdust bedding. After 2 weeks, the male was removed, and the females were housed individually. The day of delivery was considered as pnd 0 . On pnd 1, litters were culled to four males and four females.

\section{Plus-maze Apparatus and Testing (Experiments I and II)}

The elevated plus-maze (Holmes and Rodgers, 1999) had two open arms $(27 \times 5 \times 0.25 \mathrm{~cm})$ and two closed arms $(27 \times 5 \times 15 \mathrm{~cm})$ extended from a common central platform $(5 \times 5 \mathrm{~cm})$. The apparatus was made in Plexiglas (black floor, transparent walls) and was elevated to a height of $40 \mathrm{~cm}$ above the floor level. Open-arm exploration was encouraged by the inclusion of a slight raised edge $(0.25 \mathrm{~cm})$ around their perimeter.

For plus-maze testing, mice were gently placed on the central platform facing a closed arm and allowed to explore freely the entire maze for a single 6-min session. To avoid unnecessary distractions, the test was carried out under dim illumination and the experimenters were not present in the testing room. After each animal was tested, the plus-maze was thoroughly cleaned with wet and dry cloths. All sessions were recorded by a video-camera (positioned above and at approx. $60^{\circ}$ to the maze). Videotapes were then scored by a highly trained observer, blind to group assignment of animals, using an ethological software (The Observer v3.0 for DOS, Noldus Information Technology, The Netherlands). The following measures were obtained: (1) latency to enter each arm; (2) number of entries in each arm; (3) time spent in each arm.

\section{Experiment I-Spontaneous Nicotine Consumption}

Mouse pups were weaned on pnd 21, three nonsibling subjects being housed in each Plexiglas cage $(33 \times 13 \times 14 \mathrm{~cm})$, according to sex. According to a splitlitter design, three same-sex sibling animals coming from 
the same litter were randomly assigned to undergo the fluid-exposure period (12 days) during one of three different age periods, namely Pre-adolescence (pnd 2435), Mid-adolescence (pnd 37-48), or Post-adolescence (pnd 50-61). Litters were randomly assigned to either the Nicotine group $(n=9)$ or the Water group $(n=5)$.

During the specific age period of exposure, animals were adapted to restricted water availability $(2 \mathrm{~h} /$ day from 0930 to 1130) as described previously (Adriani et al, 2002). Food pellets were always available ad libitum. For the daily drinking session, animals were gently placed individually in experimental cages of the same size and shape as home cage (standard cage tops accommodated two bottles and food pellets were provided on the floor of the cage). After a 2-day acclimation period (both bottles containing water), animals within each age group underwent the fluid-exposure period (12 days). Mice from the Water group were provided with two bottles containing water, while animals from the Nicotine group had a choice between water and a nicotine solution $(10 \mathrm{mg} / \mathrm{l})$. Daily fluid and drug intake were measured for each animal (see Adriani et al, 2002). Following the 12-day fluid-exposure period, water was again available ad libitum for all animals.

On the day of their last drinking session, animals of the specific age group underwent the plus-maze test. Immediately following the end of the drinking session, animals were transported from the animal room to the testing room, left undisturbed for at least $1 \mathrm{~h}$, and then scored in the plusmaze. After the test, animals were returned to the animal room. When adults, 2 months after last nicotine exposure, all animals were tested for locomotor habituation to a novel environment in a 3-day test. Mice were transported from the animal room to the testing room, and left undisturbed for at least $1 \mathrm{~h}$ before the locomotor-habituation test.

\section{Locomotor-habituation Apparatus and Testing in Experiment I}

Locomotor activity was measured in opaque Plexiglas rectangular boxes with smooth walls and floor $(20 \times 14 \times 27 \mathrm{~cm})$. Each box was provided with four infrared photobeams, placed on the wall a few centimeter above the floor. Beam interruptions were recorded by an IBM computer equipped with a specific custom-made software, and the activity rate (number of beam interruptions per second) was measured. Mice were exposed for a 30-min session on three subsequent days. Each 30-min session was automatically subdivided into 5-min intervals. The floor of the apparatus was cleaned after each animal was tested and the test was carried out under dim illumination. This procedure allowed the assessment of intra- and intersession habituation profiles of the locomotor response.

\section{Experiment II - Experimenter-Imposed Nicotine Administration}

Mouse pups were weaned on pnd 21, four same-sex pups coming from the same litter being housed in each cage $(33 \times 13 \times 14 \mathrm{~cm})$. Separate litters $(n=5$ per group) were randomly assigned to be repeatedly treated with nicotine either during adolescence (from pnd 35 to 44) or at adulthood (pnd $>70$ ). During the specific age period, the four sibling mice within each cage were injected with nicotine $(0,0.03,0.10$, and $0.30 \mathrm{mg} / \mathrm{kg}$ i.p. per day for 10 days).

On the first day of drug treatment, animals were also tested in the plus-maze for acute nicotine effects. Animals were transported from the animal room to the testing room, left undisturbed for at least $1 \mathrm{~h}$, and then tested in the plusmaze $15 \mathrm{~min}$ after acute nicotine administration. After the plus-maze session, animals were returned to the animal room and kept in standard conditions. Then, 2 months following the 10-day nicotine treatment period, animals were killed by decapitation and brain samples were collected for neurochemical assessment.

\section{Neurochemical Assessment in Experiment II}

Brains were dissected on ice, the striatum, hippocampus, and prefrontal cortex were quickly removed from the brain and immediately stored at $-80^{\circ} \mathrm{C}$. Brain structures were weighed and homogenized in a 9 volume of homogenization buffer (50 mM Tris- $\mathrm{HCl}$, pH 7.4, 10\% sucrose, 5 mM EDTA) containing a protease-inhibitor cocktail (Sigma, USA). The homogenate was first centrifuged at $1000 \mathrm{~g}$ for $10 \mathrm{~min}$ at $4{ }^{\circ} \mathrm{C}$ to remove nuclei. Supernatants were immediately centrifuged at $34000 \mathrm{~g}$ for $20 \mathrm{~min}$ at $4{ }^{\circ} \mathrm{C}$. Then, the supernatants were discarded and the pellets were washed once, resuspended in $50 \mathrm{mM}$ Tris- $\mathrm{HCl}, \mathrm{pH} 7.4$, assayed for protein content, using modified micro Lowry assay (Bio-RAD, USA) and stored at $-80^{\circ} \mathrm{C}$ until analyzed.

We analyzed the levels of AMPA (GluR2/3) and NMDA (NR2A/B) glutamate-receptor subunits. Samples $(10 \mu \mathrm{g} /$ line) were diluted in Laemmli's samples buffer, loaded into precast $7.5 \%$ SDS-PAGE polyacrylamide (Bio-Rad, USA) gel at a quantity of $10 \mu \mathrm{g}$ of total protein per line and ran at $125 \mathrm{~V}$ for about $1.5 \mathrm{~h}$. Then proteins were transferred to

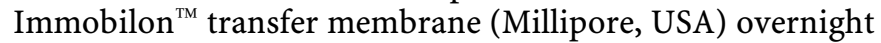
at room temperature in a Bio-Rad semidry transfer cell, using $40 \mathrm{mM}$ Tris, $39 \mathrm{mM}$ glycine, and $10 \%$ methanol as transfer buffer. Blots were blocked in 3\% Carnation not-fat dry milk, TBS (100 mM Tris, $0.9 \% \mathrm{NaCI}, \mathrm{pH} 7.6)$ for $2 \mathrm{~h}$. Following a brief wash with Tween-20 Tris-buffered saline (TTBS: $100 \mathrm{mM}$ Tris, 0.9\% NaCI, 0.05\% Tween 20, pH 7.6), blots were incubated with rabbit polyclonal antibodies diluted to a concentration of $0.2 \mu \mathrm{g} / \mathrm{ml}$ (Chemicon, USA) for $2 \mathrm{~h}$.

Blots included a range of protein standards $(5-40 \mu \mathrm{g}$ of the total solubilizate of mouse hippocampus or striatum). These standards were used to generate a protein standard curve. The linearity of the relationship between optical density and protein concentrations was verified and all samples matched to the linearity area. Rabbit anti-actin polyclonal antibodies (Chemicon, USA) were used as an additional internal control, mainly to avoid possible errors in protein concentrations. By looking at data on the actin internal standards, no evident interindividual variation emerged in sample loading within each group. Thus, actin data were not considered for correction within bands of interest.

Blots were washed three times in TTBS for $10 \mathrm{~min}$ each. Secondary antibodies (ImmunoPure, goat anti-rabbit, alkaline phosphatase conjugate, Pierce, USA) were applied for $1 \mathrm{~h}$ (dilution $1: 5000$ ) in TTBS. Blots were washed four 
times in TTBS for 10 min each and developed in 1-Step ${ }^{\text {TM }}$ NBT/BCIP substrate (Pierce, USA) during $10 \mathrm{~min}$. Blots were scanned and digitized, and densitometric analysis performed using the Scion 'Image-Pro' Software (website http://www.scioncorp.com).

\section{Drugs}

All concentrations and doses are expressed as nicotine ([-]1-methyl-2-[3-pyrityl] pyrrolidine) base. In experiment I, the drinking solutions were prepared by dissolving nicotine hydrogen tartrate (SIGMA, USA) in tap water. The drug concentration $(10 \mathrm{mg} / \mathrm{l})$ was selected on the basis of previous experience (Adriani et al, 2002). In experiment II, nicotine hydrogen tartrate was dissolved in saline (SAL, $\mathrm{NaCl} 0.9 \%)$. Doses were selected on the basis of literature data (Brioni et al, 1993; O’Neil and Brioni, 1994).

\section{Design and Data Analysis}

We adopted a split-litter design, each litter representing the statistical unit. This procedure is widely requested and adopted in studies dealing with immature animals, where sibling pups within each litter are not independent (Chiarotti et al, 1987). Separate analyses and post hoc comparisons (Tukey HSD) were performed when allowed. In experiment I, treatment was a between-litter factor, all other were within-litter factors. The general model was a 2 drug (Water $v s$ Nicotine) $\times 2$ sex (females $v s$ males) $\times 3$ age (Pre- vs Mid-vs Post-adolescents) model. For plus-maze latency measures, a two-level arm (closed $v s$ open) factor was included in the ANOVA. For the locomotor-habituation test, the Water group was compared to the Nicotine group to assess the effects of developmental nicotine exposure. The design of the experiment included a 3 days $\times 6$ repeated measures interaction. In experiment II, age was a betweenlitter factor, and all other were within-litter factors. The general model was a 2 age (adolescent $v s$ adult) $\times 2$ sex (female $v s$ male $) \times 4$ dose $(0, \quad 0.03, \quad 0.1, \quad 0.3 \mathrm{mg} / \mathrm{kg}) \times$ repeated measures on the same subject.

\section{RESULTS}

\section{Experiment I-Spontaneous Nicotine Consumption}

Nicotine consumption. During the drug-exposure period, animals belonging to Pre-, Mid- or Post-adolescence age groups showed a mean daily nicotine intake of $1.39 \pm 0.02 \mathrm{mg} / \mathrm{kg}$ (see also Adriani et al, 2002).

Latency to enter the arms. A main effect of Arm, $\mathrm{F}_{(1,12)}=48.7, p<0.01$, was found, confirming that mice entered the closed arms with a shorter latency, when compared to the open arms. Age-related changes were evident, the Age by Arm interaction, $\mathrm{F}_{(1,12)}=5.04, p<0.01$, and the Age by Arm by Drug interaction, $\mathrm{F}_{(2,24)}=5.58$, $p<0.01$, being significant. As a whole, no significant or reliable changes emerged for latencies toward closed arms, whereas huge differences characterized the open arm. In particular, as shown in Figure 1 (upper left panel), Midadolescents from the Water group entered the open arms with a significantly shorter latency than subjects from the
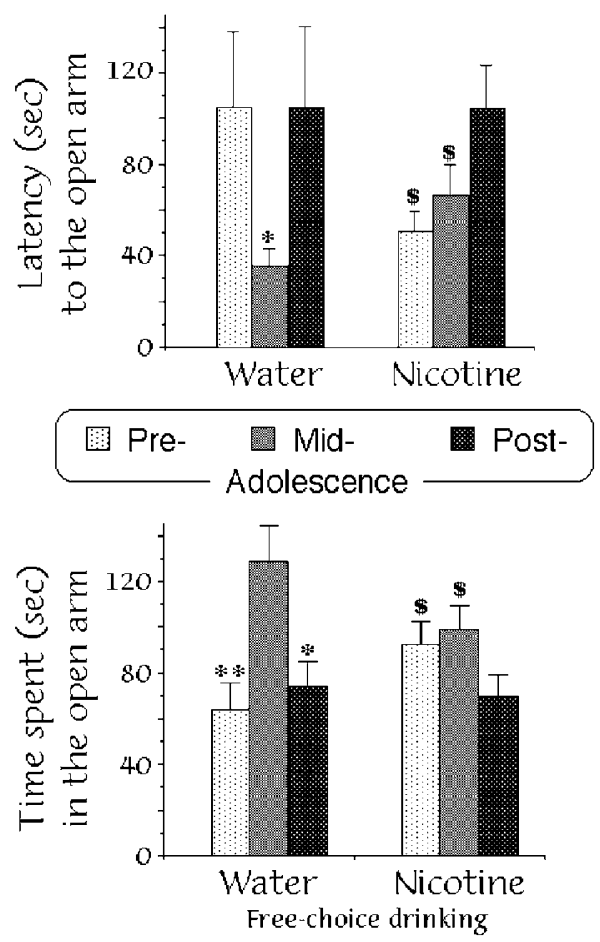

Figure I Upper panel: Mean (SEM) latency (s) to the first approach to the open arms. Lower panel: Mean (SEM) time (s) spent in the open arm (Experiment I). Mice during Pre-, Mid- or Post-adolescence underwent 12 days of free-choice drinking sessions, one bottle containing water and the other containing nicotine $(n=18)$ or water $(n=10)$. Mice were tested in the plus-maze at least I $\mathrm{h}$ after their last drinking session. In the absence of any effect of the sex variable, data from males and females have been collapsed. $* p<0.05,{ }^{*} p<0.01$ in multiple comparisons between age groups. ${ }^{\$} p<0.05$ in multiple comparisons between the Nicotine group and the Water control.

other ages $(p<0.05)$. This ontogenetic profile was profoundly altered in the Nicotine group (see Figure 1, upper right panel). In fact, Mid-adolescents that spontaneously self-administered nicotine showed a significantly higher latency to enter the open arm, when compared to their same-age controls $(p<0.05)$. Further, Pre-adolescents from the Nicotine group entered the open arm with a shorter latency than same-age controls $(p<0.05)$. The oral selfadministration of nicotine did not affect the performance in the Post-adolescent group. Such a lack of effects could possibly reflect the development of tolerance due to chronic drug exposure.

Time spent in open arms. As expected, animals spent in general about two-fold more time in the closed than in the open arms (data not shown). As for time spent in the open arm, a significant main effect of Age was found in the ANOVA, $\mathrm{F}_{(2,24)}=7.87, p<0.01$, together with a significant Age by Drug interaction, $\mathrm{F}_{(2,24)}=3.24, p<0.05$. An ontogenetic profile emerged within the Water group (see Figure 1, lower panel), with Mid-adolescents spending a larger amount of time in the unprotected area of the apparatus, when compared to animals from the other age groups $(p<0.05)$. Interestingly, age-related differences were no more evident within the Nicotine group (see Figure 1, lower panel), the amount of time spent by Mid-adolescents in the open arms of the maze being strongly reduced by the 
oral self-administration of nicotine $(p<0.05)$. Further, Preadolescents from the Nicotine group spent slightly more time in the open arm than controls $(p<0.05)$. In other words, the characteristic age-related profile, which was consistent with previous work in our lab (Macri' et al, 2002), was actually dampened in animals allowed to drink nicotine.

Percent open-arm entries. Since the number of entries into each part of the apparatus is strictly related to levels of locomotor activity (ie an animal scoring high activity rates might enter each arm more frequently than another scoring lower), the open-arm entries were divided for the total number of entries. The profile of results for this variable was nicely overlapping to that described for time spent in the open arm (data not shown). Indeed, a main effect of Age, $\mathrm{F}_{(2,24)}=7.97, p<0.01$, and an Age by Drug interaction, $\mathrm{F}_{(2,24)}=5.55, p<0.01$, was found. An elevated proportion of open-arm entries was shown by Mid-adolescents within the control Water group, when compared to younger and older subjects. Again, a dramatic reduction of this parameter was exhibited by Mid-adolescents from the Nicotine group, when compared to their same-age controls $(p<0.05)$. Hence, the regular ontogenetic profile was dramatically dampened in the Nicotine group.

Frequency of closed-arm entries. The number of closedarm entries has been suggested as the best measure for locomotor activity in the plus-maze paradigm. No reliable or significant effects of Age and Drug variables were found (data not shown).

Body weight. As expected, body weight differed depending on the sex, $\mathrm{F}_{(1,12)}=100, p<0.01$, and the age, $\mathrm{F}_{(2,24)}=61.3$, $p<0.01$, of the subjects. No carry-over effects of prior drug intake were found on this measure (data not shown).

\section{Locomotor Activity and Habituation Profiles in Experiment I}

Long-term effect of nicotine exposure during adolescence. The ANOVA yielded a main effect of Day, $\mathrm{F}_{(3,36)}=4.39$, $p<0.01$, and Time, $\mathrm{F}_{(5,60)}=8.64, p<0.01$. Specifically, adult mice showed a clearcut intra- and intersession habituation profiles (see Figure 2). Interestingly, these profiles were also a function of the developmental age of drug exposure, a significant three-way Age by Day by Time interaction, $\mathrm{F}_{(30,360)}=8.64, p<0.01$, being found. To better depict this interaction, separate analyses were performed within each age group. For Pre- and Post-adolescents, a main effect of Time, $\mathrm{F}_{(5,60)}=20.3$ and 11.9 , respectively, $p<0.01$, confirmed the presence of a clearcut within-session habituation profile. No carry-over effects of nicotine exposure emerged, although a nonsignificant tendency toward lower activity rates was apparent for mice exposed to nicotine when Postadolescents. Interestingly, within Mid-adolescents, the Drug by Day interaction, $\mathrm{F}_{(3,36)}=4.27, p<0.05$, and the Drug by Time interaction, $\mathrm{F}_{(5,60)}=8.64, p<0.01$, were significant. Specifically, in mice from the control Water group, locomotor activity decreased during the course of the session and over subsequent days. Conversely, such a

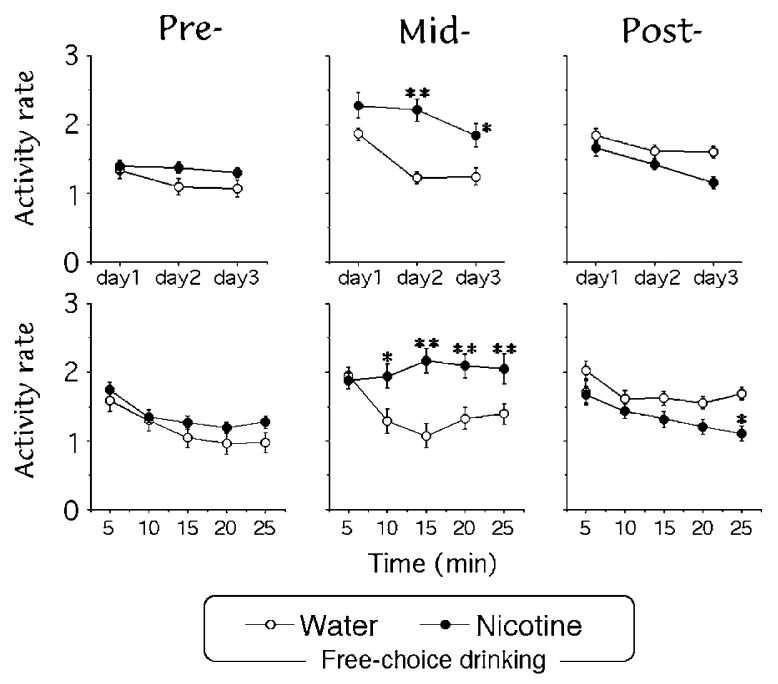

Figure 2 Mean (SEM) activity rate (number of beam interruptions per second) shown in the test for locomotor response to novelty (Experiment I). Mice during Pre-, Mid- or Post-adolescence underwent 12 days of freechoice drinking sessions, one bottle containing water and the other containing nicotine $(n=18)$ or water $(n=10)$. After a 2-month nicotinefree period, mice were tested for profiles of intersession (upper panels) and intrasession (lower panels) locomotor habituation, following exposure to a novel environment (a 30-min session for 3 days). In the absence of any effect of the sex variable, data from males and females have been collapsed. Multiple comparisons: $* p<0.05,{ }^{*} p<0.0$ l between Water and Nicotine groups.

classical habituation profile was not evident at all in adult mice that were exposed to nicotine during Mid-adolescence. Rather, the latter subjects exhibited a clear deficit of habituation and were associated with abnormally elevated activity levels, when compared to controls (see Figure 2).

As a whole, these results revealed that both the intra- and intersession habituation profiles were specifically disrupted at adulthood by nicotine exposure during Mid-adolescence. Comparable exposure during other periods of adolescence was completely devoid of a similar effect (or tended to produce an opposite effect in the case of Post-adolescents). These findings may be interpreted as evidence of a narrow window of peculiar vulnerability to nicotine, as far as its long-term sequels are concerned.

\section{Experiment II-Experimenter-imposed Nicotine Administration}

Latency to enter the open arm. Data regarding the latency to enter closed arms were not included in the analysis, with all values being very close to zero. The ANOVA yielded a main effect of Age, $\mathrm{F}_{(1,27)}=10.3, p<0.01$, indicating that adults entered the open arm with a higher latency than adolescents ( $92 \pm 13$ vs $39 \pm 8$ s, respectively). No significant or reliable effects of other variables were found.

Time spent in the open arms. As expected, time spent in the closed arms was about twice the time spent in the open arms (data not shown). As for time spent in open arms, the ANOVA was significant for the Age factor, $F_{(1,30)}=10.2$, $p<0.01$, and for the Age by Dose interaction, $\mathrm{F}(3,30)=4.02$, $p<0.05$. Specifically, adult animals injected with nicotine 
$0.10 \mathrm{mg} / \mathrm{kg}$ spent much more time in the open arms, when compared to controls (see Figure 3, left panel). Conversely, upon the same dosage, adolescent animals tended to show a reduction of time spent in the open arm. Post hoc analysis revealed a significant difference in the response to the $0.10 \mathrm{mg} / \mathrm{kg}$ dose between the two ages $(p<0.05)$. In contrast, no differences emerged at all other dosages.

Percent open arm entries. The ANOVA was significant for the Age factor, $F_{(1,30)}=10.2, p<0.01$, and for the Age by Dose interaction, $\mathrm{F}_{(3,30)}=3.56, p<0.05$. Specifically, adult animals injected with nicotine $0.10 \mathrm{mg} / \mathrm{kg}$ showed much more open arms entries, when compared to controls (see Figure 3, right panel). Conversely, upon the same dosage, adolescent animals showed a tendency toward a reduction of open arm entries. Post hoc analysis revealed a significant difference between adolescents and adults only upon the $0.10 \mathrm{mg} / \mathrm{kg}$ dose. Again, the two ages did not differ at all other doses.

Frequency of closed arm entries. The ANOVA was significant for the Age factor, $\mathrm{F}_{(1,30)}=5.35, p<0.05$, suggesting that adult animals showed much less closed arm entries, when compared to adolescents (see Table 1). However, this age-related profile was blunted by nicotine administration, since closed arm entries were somewhat increased in adolescent animals and conversely decreased in adults. Indeed, post hoc analysis confirmed a significant difference between ages only within the control SALinjected group $(p<0.05)$.

\section{Neurochemical Assessment of Glutamate Receptors in Experiment II}

Data revealed that even a short period (10 day) of nicotine administration produces long-lasting changes of immunoreactivity for AMPA glutamate receptors in the striatum and hippocampus of mice. These alterations were age- and dosedependent. As for striatum, the ANOVA revealed a main Age effect, $\mathrm{F}_{(1,15)}=8.63, p<0.01$, and a Drug by Age
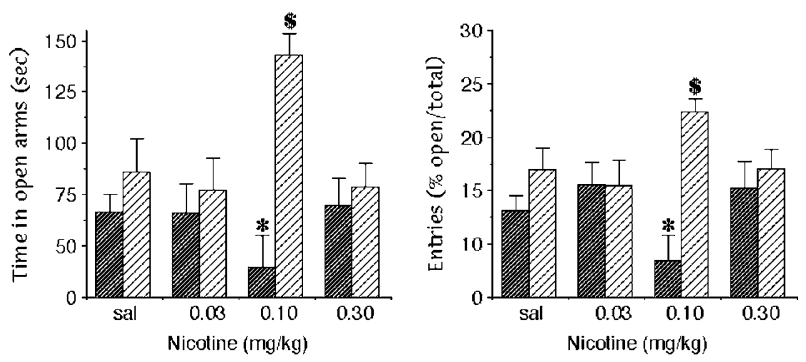

IUA Adolescents $\square$ Adults

Figure 3 Left panel: Mean (SEM) time (s) spent in the open arm. Right panel: Mean (SEM) entries (\% of total) in the open arm. Adolescent (pnd 35) and adult (pnd >70) mice were tested in the plus-maze 15 min after acute nicotine injection (Experiment II). In the absence of any effect of the sex variable, data from males and females have been collapsed $(n=10)$. $* p<0.05$ in multiple comparisons between age groups. $\$ p<0.05$ in multiple comparisons between a given nicotine dose and the SAL-injected control. interaction, $\mathrm{F}_{(3,45)}=19.5, p<0.001$. The immunoreactivity of GluR2/3 subunits of AMPA glutamate receptor dosedependently decreased in the striatum of mice exposed when adolescent (Figure 4, upper right panel), whereas in the brain of animals exposed when adult exactly the opposite effect was observed (Figure 4, lower right panel). As for hippocampus, the ANOVA revealed a main Drug effect, $\mathrm{F}_{(3,45)}=11.5, p<0.001$, and a Drug by Age interaction, $\mathrm{F}_{(3,45)}=6.48, p<0.01$. In the hippocampus of mice exposed when adolescent, GluR2/3 immunoreactivity decreased in a dose-dependent manner (Figure 4, upper left panel). Animals exposed at adulthood demonstrated no significant changes in hippocampal GluR2/3 levels (Figure 4, lower left panel).

As for the NR2A/B subunit of NMDA receptors in the striatum, the ANOVA yielded a main effect of Age, $\mathrm{F}_{(1,15)}=251, p<0.001$. At both ages, nicotine exposure induced a decrease of NR2A/B immunoreactivity for females and conversely no effect for males, Drug $\times$ Sex interactions, $F_{(3,24)}=3.63$ and $F_{(3,21)}=3.20$ for adult and adolescent exposure, $p<0.05$. However, this dose-dependent effect was similar at both ages. Hence, we may conclude that nicotine effects on NMDA receptors were gender-specific (see Table 2) but not age-dependent. Similarly, no reliable or significant differences due to sex or age of exposure were found for levels of NR2A/B immunoreactivity in the hippocampus.

Assessment of neurochemical parameters in the prefrontal cortex did not reveal any significant or reliable nicotineinduced change in either GluR2/3 or NR2A/B immunoreactivity. All these data confirm that NMDA receptors do not seem to play a significant role in age-related vulnerability to nicotine.

\section{DISCUSSION}

Data from the present study can be summarized as follows:

(1) Mice at Mid-adolescence showed low anxiety with an elevated exploratory drive, which was dampened after 12 days of spontaneous nicotine consumption. After 2 months, the regular profile of locomotor habituation in response to novelty was markedly disrupted by nicotine exposure. This change was specific for Mid-adolescent animals.

Table I Mean $( \pm$ SEM) Frequency of Closed-arm Entries in the Plus-maze, as a Function of Age and Acute Nicotine Treatment in Experiment II

\begin{tabular}{lcc}
$\begin{array}{l}\text { Nicotine dose } \\
\text { (mg/kg) }\end{array}$ & $\begin{array}{c}\text { Adolescents } \\
\text { (pnd 35) }\end{array}$ & $\begin{array}{c}\text { Adults } \\
\text { (pnd }>\mathbf{7 0})\end{array}$ \\
\hline SAL & $22.3 \pm 2.0 *$ & $11.9 \pm 1.0$ \\
NIC 0.03 & $16.4 \pm 0.9$ & $15.9 \pm 2.0$ \\
NIC 0.10 & $18.0 \pm 2.3$ & $16.8 \pm 1.4$ \\
NIC 0.30 & $18.1 \pm 1.1$ & $15.3 \pm 1.4$ \\
\hline
\end{tabular}

In the absence of significant or reliable gender differences, the two sexes have been pooled $(n=10)$. ${ }^{*} p<0.05$ in post hoc comparisons between ages. 

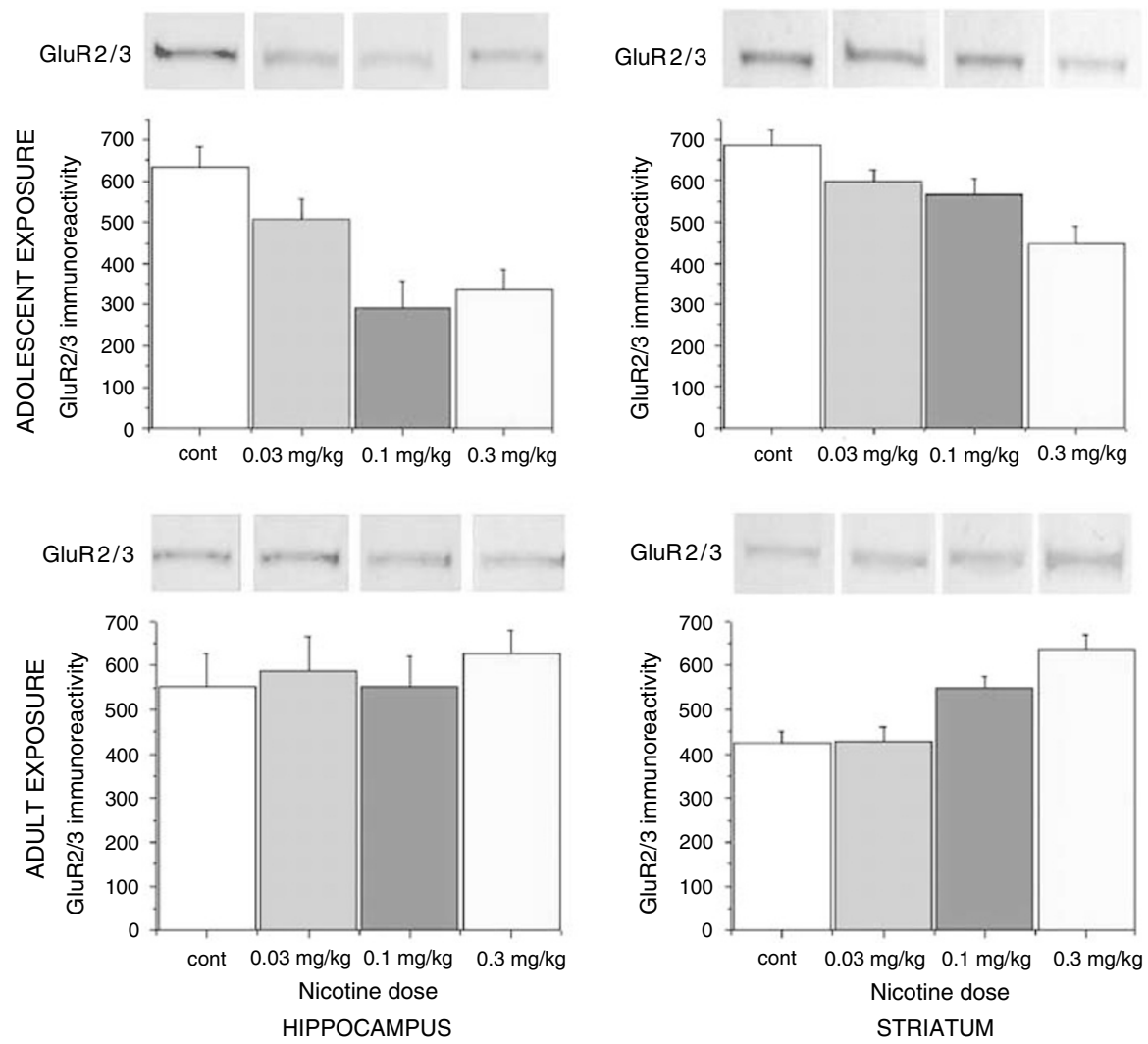

Figure 4 Optical density of the GluR2/3 subunit of AMPA receptors in the Western blots from striatum or hippocampus. Mice underwent 10 days of nicotine treatment during Mid-adolescence (pnd 35-44) or when adult (pnd > 70). Animals were then killed 2 months after last drug injection (Experiment II). In the absence of any effect of the sex variable, data from males and females have been collapsed $(n=10)$.

Table 2 Mean ( \pm SEM) Optical Density of the NR2A/B Subunit of NMDA Receptors in the Western Blots from Striatum of Mice from Experiment II

\begin{tabular}{llc}
\hline Nicotine dose $\mathbf{( m g / k g )}$ & Females & Males \\
\hline SAL & $620 \pm 47$ & $672 \pm 47$ \\
NIC 0.03 & $497 \pm 58$ & $520 \pm 81$ \\
NIC 0.10 & $337 \pm 40 \$$ & $589 \pm 37 *$ \\
NIC 0.30 & $280 \pm 56 \$$ & $564 \pm 61 *$ \\
\hline
\end{tabular}

The two ages have been pooled $(n=10)$. $* 0.05$ in post hoc comparisons between sexes. $\$ p<0.05$ in post hoc comparisons between drug-exposed group and saline control.

(2) Acute nicotine administration (at the dose of $0.10 \mathrm{mg} /$ $\mathrm{kg}$ ) reduced anxiety in adults, whereas it tended to have an opposite effect in adolescent animals. A dosedependent reduction of GluR2/3 immunoreactivity was found in the striatum and hippocampus 2 months after a pretreatment with nicotine during Mid-adolescence. A comparable pretreatment, but given in already adult subjects, dose-dependently increased striatal GluR2/3 receptors and had no effect in the hippocampus.

The present study was designed to characterize the spontaneous behavior expressed by mice at different phases of adolescence, as well as the effects of nicotine. The plus- maze test can provide information not only on anxiety (Brioni et al, 1993; O'Neil and Brioni, 1994; File et al, 1998) but also on the motivation to explore despite risky conditions and hence on risk-taking behavior (Holmes and Rodgers, 1999; Macri' et al, 2002). Classically, a clearcut avoidance for the open arms is reported at adulthood for both rats and mice (File et al, 1998; Holmes and Rodgers, 1999). Interestingly, an age-related discontinuity was revealed in Experiment I. Namely, mice showed elevated preference for the open and unprotected areas at Midadolescence (pnd 48), when compared to younger (pnd 35) and older (pnd 61) ages. Accordingly, these two latter age groups did not show differences when tested in Experiment II. Data from these two experiments confirm previous findings, suggesting an enhanced exploratory drive and/or reduced anxiety associated with risky situations during Mid-adolescence (Macri' et al, 2002; see Laviola et al, 2003).

Consistent with other reports in mice (Brioni et al, 1993; O'Neil and Brioni, 1994), the acute administration of nicotine (at the specific dose of $0.10 \mathrm{mg} / \mathrm{kg}$ ) reduced anxiety in adults. Conversely, an opposite (ie anxiogenic) effect tended to appear in 35-day-old adolescent animals following acute nicotine administration (experiment II). In apparent contrast with a recent report in rats (Cheeta et al, 2001), no reliable sex differences apeared in the present mouse study. This discrepancy may perhaps be accounted for by a number of factors, including the animal species and experimental procedure. A prolonged 12-day period of spontaneous nicotine consumption during Mid-adolescence 
strongly reduced the time spent in the open and unprotected areas of the plus-maze (experiment I). Noteworthy is the fact that the ontogenetic profile of Mid- adolescents, consisting of high exploratory drive and low anxiety, was abated by nicotine consumption. In other words, the possibility to self-administer nicotine during the peculiar phase of adolescence (otherwise characterized by noveltyseeking and risk-taking behaviors; see Laviola et al, 2003) produced more anxious animals, less prone to take risks. Due to nicotine drinking, Mid-adolescent mice possibly experienced an altered development of coping strategies toward novelty and risk, and this could lead to more persistent behavioral changes into adulthood.

In fact, the regular response of adult mice to novelty (ie locomotor habituation) was markedly disrupted, when measured 2 months after spontaneous nicotine consumption during Mid-adolescence. The consequences of nicotine exposure were highly age-specific, since nicotine exposure did not affect habituation in subjects consuming nicotine at younger or older ages. Rather, the effect (if any) of later drug exposure was in the opposite direction. Enhanced locomotor response to novelty is a largely validated index of vulnerability to the addictive properties of psychostimulants (Wise and Bozarth, 1987; Piazza et al, 1991). The present findings suggest that spontaneous consumption of nicotine during $M i d$-adolescence might be associated with a drug-vulnerable phenotype at adulthood. This would be highly consistent with very recent findings obtained in rats, in that nicotine exposure during adolescence was responsible for an increased vulnerability to develop nicotine addiction (Adriani et al, 2003), when compared to comparable exposure at adulthood. Altogether, these data obtained in animal models support the epidemiological reports of increased risk toward addiction in early-onset smokers (Taioli and Wynder, 1991; Breslau and Peterson 1996).

\section{Nicotine Effects on Glutamate-receptor Subunits}

In the present study, we demonstrated that even a short period of nicotine administration drastically changed the glutamate-receptor immunoreactivity in the striatum and hippocampus of mice, when measured 2 months later. As for NMDA receptors, long-term nicotine effects were sexually dimorphic, but no age-related differences were found. It is well known that the behavioral and neurochemical effects of NMDA-pathway activation differ between sexes (D'Souza et al, 1999). In agreement with the present data, a prolonged treatment with nicotine is reported to decrease NMDA-receptor density, but only under certain conditions of NMDA-receptor activation (Zhang et al, 1994). Hence, differences in basal function of NMDA system in males and females may perhaps account for the present data.

As for AMPA receptors, nicotine-induced alterations interacted with age of exposure and were dose-dependent. Specifically, a repeated nicotine treatment during adolescence was found to reduce striatal as well as hippocampal levels of the GluR2/3 subunit of the AMPA glutamate receptor. Conversely, comparable nicotine treatment, but given when mice were adult, had no effect or enhanced these levels. These results may provide an insight into the neurobiological mechanisms responsible for adolescents' vulnerability to addictive drugs. It is important to note that adolescent and adult mice had opposite patterns of GluR2/3 immunoreactivity in response to nicotine administration. These observations may prove the existence of different molecular mechanisms leading to opposite effects on the risk of developing nicotine addiction for adolescent and adult animals.

There is some evidence in the literature that glutamatergic pathways are involved in nicotine's mechanism of action (Wise, 1996; Mansvelder and McGehee, 2002). The focal in vivo administration of an NMDA-receptor antagonist within the VTA inhibits the nicotine-induced increases of DA release within the ventral striatum, suggesting nicotinic modulation of glutamatergic transmission (Schilstrom et al, 1998). AMPA and NMDA receptors modulate the locomotor activity induced by amphetamine in mice (Burns et al, 1994). Interestingly, AMPA glutamate receptors seem to be specifically involved in drug-seeking and vulnerability to drug addiction, in that increased levels of AMPA-receptor subunits were correlated to extinction during self-administration experiments (Sutton et al, 2003). In particular, the overexpression of AMPA-receptor subunits in the ventral striatum facilitates the extinction of cocaine-seeking responses, and conversely extinction from chronic cocaine self-administration induces experience-dependent increases in the subunits of AMPA receptors in the ventral striatum (Sutton et al, 2003). Taking into account these evidences, it seems possible to suggest that nicotine exposure may affect drug-reward and addictive habit-forming processes by modulating the striatal level of AMPA receptors. In other words, the glutamatergic control over synaptic plasticity within the striatum might be strongly involved in the development of addictive habits and compulsive-intake behaviors (Winder et al, 2002; Fasano and Brambilla, 2002; Gerdeman et al, 2003). The latter have been strongly involved in the development of addiction (Nestler, 2001, 2002; Robbins and Everitt, 2002). Noteworthy is the fact that mice exposed to nicotine during Mid-adolescence or when they were already adult demonstrate quite an opposite picture for AMPA immunoreactivity and hence in terms of behavioral vulnerability.

\section{CONCLUSION}

Addiction is thought to involve persistent neurobiological changes that facilitate relapse to drug use despite efforts to abstain. Epidemiological evidence warned us that a greater risk of developing addiction is associated with an early onset of drug use (see Introduction). However, the etiopathological mechanisms are so far poorly understood. Nicotine exposure is well known to produce long-term consequences on forebrain systems (for a review, see Balfour et al, 1998). Yet, only recently a growing interest became evident about the fact that neurobehavioral effects of nicotine exposure during adolescence differ from comparable exposure in adult subjects (Trauth et al, 1999, 2001; Adriani et al, 2003).

The present results provide a neurobiological basis for increased vulnerability to nicotine in the still plastic brain of adolescents. We found: (1) a specific age window for 
nicotine-induced disruption of the locomotor response to novelty (a validated index of enhanced vulnerability to drugs); and (2) an opposite pattern of dose-dependent changes in glutamate-receptor immunoreactivity as a consequence of nicotine exposure during either adolescence or adulthood. According to literature (DiCiano and Everitt, 2001; DiCiano et al, 2001; Sutton et al, 2003), the up- or downregulation of AMPA receptors may suggest a better or worsened control, respectively, over pavlovian approach to drug-conditioned cues and over drug-seeking behavior. Nicotine pretreatment in Mid-adolescent mice, but not comparable exposure at adulthood, produced a profound and long-lasting downregulation of the AMPA-receptor pathway. This change could alter intrastriatal plasticity and habit-forming processes (Winder et al, 2002; Fasano and Brambilla, 2002; Gerdeman et al, 2003), which have been involved in potential vulnerability to addiction (Nestler, 2001, 2002; Robbins and Everitt, 2002).

In summary, the developing brain of adolescent seems to be highly vulnerable to nicotine (Slotkin, 2002). It appears that Mid-adolescent mice (and rats) undergo a specifically 'vulnerable' age period and may represent a suitable animal model for human adolescence, with enough face and construct validity (Spear, 2000), being able to show (1) behavioral features that resemble those found in human adolescents; (2) an elevated vulnerability to the consumption of drugs, compared to other ages; and (3) marked longterm vulnerability as a consequence of such exposure.

\section{ACKNOWLEDGEMENTS}

This study was supported by the Research Project on 'Psychobiological risk or protection factors... during development' (intramural grant to GL), Istituto Superiore di Sanita', Rome, and by the Collaborative Linkage Grant No. 978219 (to GL and SD), NATO, Brussels. We wish to thank Angelina Valanzano for expert technical assistance.

\section{REFERENCES}

Adriani W, Chiarotti F, Laviola G (1998). Elevated novelty seeking and peculiar D-amphetamine sensitization in periadolescent mice compared to adult mice. Behav Neurosci 112: 1152-1166.

Adriani W, Laviola G (2000). A unique profile of behavioral and hormonal hyporesponsivity in periadolescent mice. Neuropharmacology 39: 334-346.

Adriani W, Laviola G (2003). Elevated levels of impulsivity and reduced place conditioning with $\mathrm{D}$-amphetamine: two behavioral features of adolescence in mice. Behav Neurosci 117: 695-703.

Adriani W, Macri S, Pacifici R, Laviola G (2002). Peculiar vulnerability to nicotine oral self-administration in mice during early adolescence. Neuropsychopharmacology 27: 212-224.

Adriani W, Spijker S, Deroche-Gamonet V, Laviola G, Le Moal M, Smit $\mathrm{AB}$ et al (2003). Evidence for enhanced neurobehavioral vulnerability to nicotine during periadolescence in rats. $J$ Neurosci 23: 4712-4716.

Anthony JC, Petronis KR (1995). Early-onset drug use and risk of later drug problems. Drug Alcohol Dependence 40: 9-15.

Arnett J (1992). Reckless behavior in adolescence: a developmental perspective. Dev Rev 12: 339-373.

Balfour DJK, Benwell MEM, Birrell CE, Kelly RJ, Al-Aloul M (1998). Sensitization of the mesoaccumbens dopamine response to nicotine. Pharmacol Biochem Behav 59: 1021-1030.
Breslau N, Peterson EL (1996). Smoking cessation in young adults: age at initiation of cigarette smoking and other suspected influences. Am J Public Health 86: 214-220.

Brioni JD, O'Neill AB, Kim DJB, Decker MW (1993). Nicotinic receptor agonists exhibit anxiolytic-like effects on the elevated plus-maze test. Eur J Pharmacol 238: 1-8.

Burns LH, Everitt BJ, Kelley AE, Robbins TW (1994). Glutamatedopamine interactions in the ventral striatum: role in locomotor activity and responding with conditioned reinforcement. Psychopharmacology 115: 516-528.

Cheeta S, Irvine EE, Tucci S, Sandhu J, File SE (2001). In adolescence, female rats are more sensitive to the anxiolytic effect of nicotine than are male rats. Neuropsychopharmacology 25: 601-607.

Chiarotti F, Alleva E, Bignami G (1987). Problems of test choice and data analysis in behavioral teratology: the case of prenatal benzodiazepines. Neurobehav Toxicol Teratol 9: 179-186.

Dajas-Bailador FA, Lima PA, Wonnacott S (2000). The a7 nicotinic acetylcholine receptor subtype mediates nicotine protection against NMDA excitotoxicity in primary hippocampal cultures through a $\mathrm{Ca}^{2+}$ dependent mechanism. Neuropharmacology 39: 2799-2807.

DiCiano P, Cardinal RN, Cowell RA, Little SJ, Everitt BJ (2001). Differential involvment of NMDA, AMPA/kainate, and dopamine receptors in the nucleus accumbens core in the acquisition and performance of pavlovian approach behavior. J Neurosci 21: 9471-9477.

DiCiano P, Everitt BJ (2001). Dissociable effects of antagonism of NMDA and AMPA/KA receptors in the nucleus accumbens core and shell on cocaine-seeking behavior. Neuropsychopharmacology 25: 341-360.

DiFranza JR, Rigotti NA, McNeill AD, Ockene JK, Savageau JA StCyr D, Coleman M (2000). Initial symptoms of nicotine dependence in adolescents. Tobacco Control 9: 313-319.

D'Souza DN, Harlan RE, Garcia MM (1999). Sexual dimorphism in the response to $N$-methyl-D-aspartate receptor antagonists and morphine on behavior and c-Fos induction in the rat brain. Neuroscience 93: 1539-1547.

Faraday MM, Elliott BM, Grunberg NE (2001). Adult vs adolescent rats differ in biobehavioral responses to chronic nicotine administration. Pharmacol Biochem Behav 70: 475-489.

Fasano S, Brambilla R (2002). Cellular mechanisms of striatumdependent behavioral plasticity and drug addiction. Curr Mol Med 2: 649-665.

Ferris CF, Shtiegman K, King JA (1998). Voluntary ethanol consumption in male adolescent hamsters increases testosterone and aggression. Physiol Behav 63: 739-744.

File SE, Kenny PJ, Ouagazzal AM (1998). Anxiolytic and anxiogenic effects of nicotine in the social interaction test: role of dorsal hippocampus. Behav Neurosci 112: 1423-1429.

Gerdeman GL, Partridge JG, Lupica CR, Lovinger DM (2003). It could be habit forming: drugs of abuse and striatal synaptic plasticity. Trends Neurosci 26: 184-192.

Holmes A, Rodgers RJ (1999). Influence of spatial and temporal manipulations on the anxiolytic efficacy of chlordiazepoxide in mice previously exposed to the elevated plus-maze. Neurosci Biobehav Rev 23: 971-980.

Kandel DB, Yamaguchi K, Chen K (1992). Stages of progression in drug involvement from adolescence to adulthood: further evidence for the gateway drug theory. J Study Alcohol 53: 447-457.

Laviola G, Macri' S, Morley-Fletcher S, Adriani W (2003). Risktaking behavior in adolescent mice: psychobiological determinants and early epigenetic influence. Neurosci Biobehav Rev 27: 19-31.

Macri' S, Adriani W, Chiarotti F, Laviola G (2002). Risk-taking during exploration of a plus-maze is greater in adolescent than in juvenile or adult mice. Anim Behav 64: 541-546. 
Mansvelder HD, McGehee D (2002). Cellular and synaptic mechanisms of nicotine addiction. J Neurobiol 53: 606-617.

Meyer EL, Gahring LC, Rogers SW (2002). Nicotine preconditioning antagonizes activity-dependent caspase proteolysis of a glutamate receptor. J Biol Chem 277: 10869-10875.

Nestler EJ (2001). Molecular basis of long-term plasticity underlying addiction. Nat Rev Neurosci 2: 119-128.

Nestler EJ (2002). Common molecular and cellular substrates of addiction and memory. Neurobiol Learn Memory 78: 637-647.

O’Neil AB, Brioni JD (1994). Benzodiazepine receptor mediation of the anxiolitic-like effect of (-)-nicotine in mice. Pharmacol Biochem Behav 49: 755-757.

Piazza PV, Maccari S, Deminiere JM, Le Moal M, Mormede P, Simon H (1991). Corticosterone levels determine individual vulnerability to amphetamine self-administration. Proc Natl Acad Sci USA 88: 2088-2092.

Picciotto MR, Zoli M, Rimondini R, Lena C, Marubio LM, Pich EM et al (1998). Acetylcholine receptors containing the beta 2 subunit are involved in the reinforcing properties of nicotine. Nature 391: 173-177.

Prendergast MA, Harris BR, Mayer S, Holey RC, Hauser KF, Littleton JM (2001). Chronic nicotine exposure reduces NMDA receptor-mediated damage in the hippocampus without altering calcium accumulation or extrusion: evidence of calbindin-D28K overexpression. Neuroscience 102: 75-85.

Robbins TW, Everitt BJ (2002). Limbic-striatal memory systems and drug addiction. Neurobiol Learn Memory 78: 625-636.

Robins LN, Przybeck TR (1985). Age of onset of drug use as a factor in drug and other disorders. In: Jones CL, Battjes RL (eds). Etiology of Drug Abuse: Implications for Prevention, NIDA Research Monograph, NIH publication: Rockville, MD, Vol 56, 178-192.

Schilstrom B, Nomikos GG, Nisell M, Hertel P, Svensson TH (1998). $N$-methyl-D-aspartate receptor antagonism in the ventral tegmental area diminishes the systemic nicotine-induced dopamine release in the nucleus accumbens. Neuroscience 82: 781-789.

Slotkin TA (2002). Nicotine and the adolescent brain: insights from an animal model. Neurotoxicol Teratol 24: 369-384.

Spear LP (2000). The adolescent brain and age-related behavioral manifestations. Neurosci Biobehav Rev 24: 417-463.
Spear LP, Brake SC (1983). Periadolescence: age-dependent behavior and psycho-pharmacological responsivity in rats. Dev Psychobiol 16: 83-109.

Stamford JA (1989). Development and ageing of the rat nigrostriatal dopamine system studied with fast cyclic voltammetry. J Neurochem 52: 1582-1589.

Sutton MA, Schmidt EF, Chol KH, Schad CA, Whisler K, Simmons $\mathrm{D}$ et al (2003). Extinction-induced upregulation in AMPA receptots reduces cocaine-seeking behaviour. Nature 421: 70-75.

Taioli E, Wynder EL (1991). The importance of age of starting smoking. $N$ Engl J Med 325: 968-969.

Teicher MH, Andersen SL, Hostetter JC (1995). Evidence for dopamine receptor pruning between adolescence and adulthood in striatum but not nucleus accumbens. Dev Brain Res 89: 167-172.

Torabi MR, Bailey WJ, Majd-Jabbari M (1993). Cigarette smoking as a predictor of alcohol and other drug use by children and adolescents: evidence of the 'gateway' drug effect. J Health 63: 302-306.

Trauth JA, Seidler FJ, Ali SF, Slotkin TA (2001). Adolescent nicotine exposure produces immediate and long-term changes in CNS noradrenergic and dopaminergic function. Brain Res 892: 269-280.

Trauth JA, Seidler FJ, McCook EC, Slotkin TA (1999). Adolescent nicotine exposure causes persistent upregulation of nicotinic cholinergic receptors in rat brain regions. Brain Res 851: 9-19.

Wills TA, Vaccaro D, McNamara G (1994). Novelty seeking, risk taking, and related constructs as predictors of adolescent substance use: an application of Cloninger's theory. J Subst Abuse 6: 1-20.

Winder DG, Egli RE, Schramm NL, Matthews RT (2002). Synaptic plasticity in drug reward circuitry. Curr Mol Med 2: 667-676.

Wise RA (1996). Addictive drugs and brain stimulation reward. Annu Rev Neurosci 19: 319-340.

Wise RA, Bozarth MA (1987). A psychomotor stimulant theory of addiction. Psychol Rev 94: 469-492.

Zhang X, Gong ZH, Nordberg A (1994). Effects of chronic treatment with $(+)$ - and (-)-nicotine on nicotinic acetylcholine receptors and $N$-methyl-D-aspartate receptors in rat brain. Brain Res 644: 32-39. 